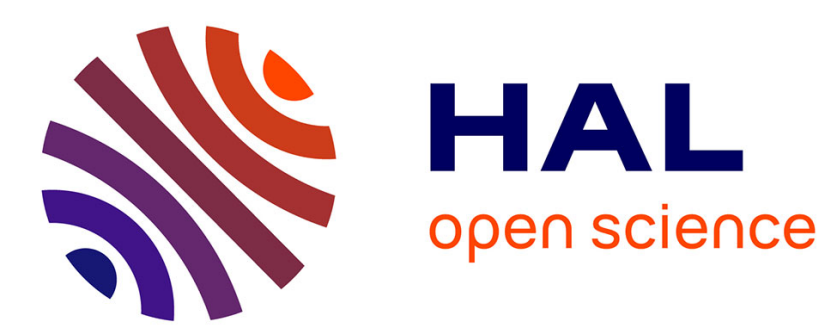

\title{
Caractérisation des verres GexSe1-x: propriétés optiques et structure locale
}

\author{
M. Bensoussan
}

\section{To cite this version:}

M. Bensoussan. Caractérisation des verres GexSe1-x: propriétés optiques et structure locale. Revue de Physique Appliquée, 1977, 12 (5), pp.753-758. 10.1051/rphysap:01977001205075300 . jpa-00244236

\section{HAL Id: jpa-00244236 https://hal.science/jpa-00244236}

Submitted on 1 Jan 1977

HAL is a multi-disciplinary open access archive for the deposit and dissemination of scientific research documents, whether they are published or not. The documents may come from teaching and research institutions in France or abroad, or from public or private research centers.
L'archive ouverte pluridisciplinaire HAL, est destinée au dépôt et à la diffusion de documents scientifiques de niveau recherche, publiés ou non, émanant des établissements d'enseignement et de recherche français ou étrangers, des laboratoires publics ou privés. 


\title{
CARACTÉRISATION DES VERRES $\mathrm{Ge}_{x} \mathrm{Se}_{1-x}$ : PROPRIÉTÉS OPTIQUES ET STRUCTURE LOCALE
}

\author{
M. BENSOUSSAN \\ Centre National d'Etudes des Télécommunications, 196, rue de Paris, 92220 Bagneux, France
}

\begin{abstract}
Résumé. - Nous présentons des résultats de mesures de l'indice de réfraction et de la limite d'absorption des verres $\mathrm{Ge}_{x} \mathrm{Se}_{1-x}$ de composition comprise entre le sélénium pur et $\mathrm{GeSe}_{2}$. Il apparaît d'après ces mesures une étroite corrélation des variations du coefficient d'absorption, de l'indice de réfraction avec $x$ et celle du spectre Raman avec ce même paramètre. Ce résultat est interprété par référence aux types de liaison possible dans ces composés.
\end{abstract}

\begin{abstract}
Index of refraction and optical absorption edge measurments, on $\mathrm{Ge}_{x} \mathrm{Se}_{1-x}$ glasses of a composition ranging from pure sélénium to $\mathrm{GeSe}_{2}$, are reported. The variations of the optical absorption coefficient and of the index of refraction with $x$ are strongly correlated to those of the Raman spectrum with the same parameter. This result is interpreted refering to the possible types of chemical bond in this compounds.
\end{abstract}

Introduction. - Le système $\mathrm{Ge}_{x} \mathrm{Se}_{1-x}$ permet la formation de composés vitreux à l'état massif sur une large plage de compositions allant de $x=0$ à $x=0,42$ [1]. Cette caractéristique semble particulièrement intéressante pour essayer de dégager des propriétés générales sur un système désordonné à partir de mesures systématiques en fonction de paramètre bien contrôlé qu'est la composition. Dans cet esprit la connaissance de l'ordre local est nécessaire non seulement pour donner une représentation de la configuration du réseau mais également pour interpréter les résultats de toute mesure physique.

De nombreux auteurs [2] ont étudié la structure locale dans une série de composés $\mathrm{Ge}_{x} \mathrm{Se}_{1-x}$ par diverses techniques expérimentales (diffraction de rayon $X$, d'électrons etc...). Il se dégage de toutes ces études un accord sur l'existence d'un environnement tétraédrique au niveau de l'atome de germanium pour $0<x<0,40$, et sur la similitude de l'environnement local dans $\mathrm{GeSe}_{2}$ amorphe et cristallin (coordinance des atomes et distances interatomiques). Cependant tous ces renseignements restent partiels notamment en ce qui concerne l'existence ou non de liaison Ge-Ge, la configuration des différents sites, et donc ne permettent pas de donner une représentation du réseau dans ces composés.

Plus récemment un modèle de structure atomique locale et de son évolution avec la teneur en germanium pour $0 \leqslant x \leqslant 0,33$ a pu être établi à partir de mesures de spectroscopie Raman [1]. L'étude de l'indice de réfraction et de la limite d'absorption permet d'étayer le modèle ainsi proposé. Nous commencerons dans la première partie par l'étude de l'indice de réfraction. La deuxième partie est consacrée à l'étude de la limite d'absorption. Dans chacune des parties nous donnerons le mode opératoire utilisé pour les mesures, les résultats obtenus et leur analyse.
1. Indice de réfraction. - 1.1 MODE OPÉRATOIRE. - L'indice de réfraction $n$ des composés vitreux $\mathrm{Ge}_{x} \mathrm{Se}_{1-x}$ pour $0 \leqslant x \leqslant 0,30$ est mesuré à $1,06 \mu$ à l'aide de prismes taillés dans ces matériaux en utilisant la méthode du minimum de déviation [3]. Afin d'éviter une réflexion totale, à cause des indices élevés dans ces matériaux, les prismes ont un angle au sommet $A$ petit (voisin de $13^{\circ}$ ). La mesure de l'angle $D$ de déviation minimum en lumière monochromatique permet de déduire l'indice de réfraction de la relation suivante :

$$
n=\frac{\sin \frac{1}{2}(A+D)}{\sin \frac{1}{2} A}
$$

La précision relative obtenue par cette méthode est de l'ordre de $0,5 \%$.

1.2 RÉSUltATS EXPÉRIMENTAUX. - Les valeurs de l'indice de réfraction sont regroupées dans le tableau I. On remarque que la variation de $n$ entre le sélénium et $\mathrm{GeSe}_{2}$ est très faible $(\Delta n / n \simeq 4 \%$. D'autre part

\section{TABLEAU I}

Indices de réfraction des composés $\mathrm{Ge}_{x} \mathrm{Se}_{1-x}$ $\grave{a} \lambda=1,06 \mu$ et $T=300 \mathrm{~K}$

$\begin{array}{lllcc}x & n & d_{\mathrm{g} / \mathrm{cm}^{2}}^{*} & \frac{1}{d} \frac{n^{2}-1}{n^{2}+2} & \frac{1}{d}\left(n^{2}-1\right) \\ \overline{0} & 2, \overline{511} & 4, \overline{28} & 0, \overline{149} 3 & 1, \overline{238} \\ 0,04 & 2,513 & 4,32 & 0,1479 & 1,227 \\ 0,15 & 2,484 & 4,360 & 0,1454 & 1,193 \\ 0,20 & 2,460 & 4,365 & 0,1436 & 1,157 \\ 0,25 & 2,452 & 4,35 & 0,1437 & 1,150 \\ 0,30 & 2,404 & 4,30 & 0,1433 & 1,116 \\ 0,33 & & 4,26 & & \end{array}$

(*) Réf. [6]. 
l'indice a tendance à décroître quand on passe de Se à $\mathrm{GeSe}_{2}$. A partir de ces mesures la valeur extrapolée de l'indice à $1,06 \mu$ pour $\mathrm{GeSe}_{2}$ amorphe est de l'ordre de $2,40 \pm 0,05$.

1.3 Analyse des Résultats. - Pour tenter d'exploiter ces résultats et en déduire des renseignements sur la structure locale dans ces composés nous avons utilisé la relation de Lorentz-Lorenz [4] :

$$
\frac{1}{d} \frac{n^{2}-1}{n^{2}+2}=\frac{R}{M}
$$

$d$ est la densité du matériau

$M$ est la masse moléculaire du composé

$R$ est la réfractivité moléculaire du composé.

De façon générale pour un composé donné $R$ est égale à la somme des réfractivités atomiques des constituants du composé ou plus exactement des unités structurales qui forment le réseau.

Cette relation tient compte d'un champ local dû à la polarisation de l'environnement local par l'onde incidente. Si l'on néglige ce champ local la relation précédente devient :

$$
\frac{1}{d}\left(n^{2}-1\right)=\frac{R}{M}
$$

$d, M$ et $R$ ont la même signification que précédemment.

Les valeurs de densités en fonction de $x$ portées dans le tableau I sont des résultats de mesures donnés dans la référence [6].

Nous avons porté sur un même graphique

$$
\frac{1}{d} \frac{n^{2}-1}{n^{2}+2} \quad \text { et } \quad \frac{1}{d}\left(n^{2}-1\right)
$$

en fonction de $x$ (Fig. 1).

La dépendance linéaire en fonction de $x$ (Fig. 1), aux erreurs expérimentales près, de $1 / d\left(n^{2}-1\right)$ montre que l'on peut décrire les composés $\mathrm{Ge}_{x} \mathrm{Se}_{1-x}$ pour $0 \leqslant x \leqslant 0,33$ par deux unités structurales apparentées aux deux composés extrêmes Se et $\mathrm{GeSe}_{2}$.

Par contre la dépendance en fonction de $x$ de $\frac{1}{d} \frac{n^{2}-1}{n^{2}+2}$ (Fig. 1) indique une cassure très nette pour $x=0,20$. Cette cassure suggère une rupture dans la nature des unités structurales.

Ce dernier résultat est plus satisfaisant que le premier car il est en accord avec ce qui a été établi à partir des résultats de la diffusion Raman dans ces composés [1]. Les atomes de germanium ont tendance à se diluer au maximum dans le sélénium et pour la composition $x=0,2$ on a des groupements $\mathrm{GeSe}_{4}$ liés entre eux par des liaisons Se-Ge ce qui ne laisse plus, à partir de cette composition, d'atomes sélénium disponibles pour former des séquences Se-Se-Se.

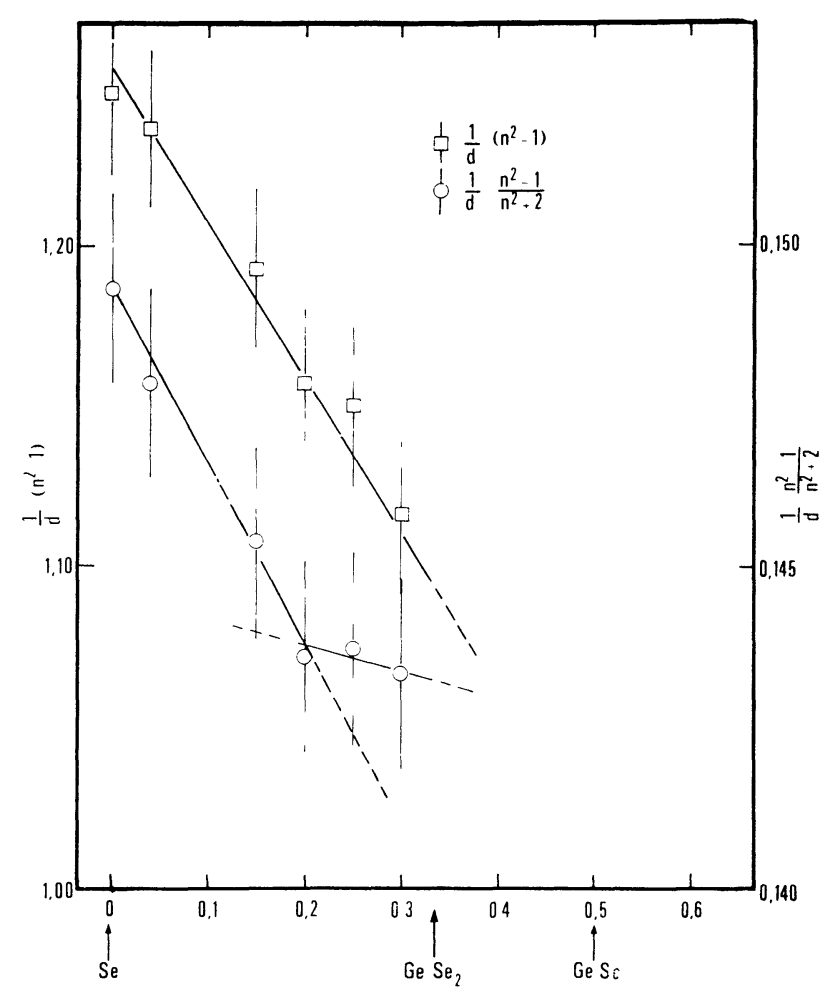

FIG. 1. - Courbes mettant en évidence une corrélation entre l'indice de réfraction $n$, la densité $d$ et la composition des composés amorphes $\mathrm{Ge}_{x} \mathrm{Se}_{1-x}(0 \leqslant x \leqslant 1 / 3)$. En fonction de la composition : (1) $\frac{1}{d}\left(n^{2}-1\right)$ est linéaire ; (2) $\frac{1}{d} \frac{n^{2}-1}{n^{2}+2}$ fait apparaître deux domaines pour $x: 0 \leqslant x \leqslant 0,20$ et $0,20 \leqslant x \leqslant 1 / 3$.

2. Limites d'absorption. - 2.1 MODE OPÉRATOIRE. - Le coefficient d'absorption $\alpha$ est déduit de la mesure de la transparence $T$ d'un échantillon d'épaisseur d par la relation (7) :

$$
T=\frac{I}{I_{0}}=\frac{(1-R)^{2} \exp -\alpha d}{1-R^{2} \exp -2 \alpha d}
$$

$I_{0}$, intensité du faisceau incident et $I$, intensité du faisceau transmis, sont mesurées successivement sur une gamme spectrale donnée (spectromètre monofaisceau). Un programme a été mis au point pour obtenir directement $\alpha$ sur ordinateur, à partir des valeurs de $I$ et $I_{0}$.

Nous avons pris le coefficient de réflexion $R$ constant et indépendant de la longueur d'onde. Compte tenu des valeurs d'indices rapportées précédemment nous avons adopté $R=0,2$ pour les composés vitreux quel que soit $x$. Il faut noter que, d'une façon générale, la valeur du coefficient de réflexion utilisée à quelques pour cents près n'est pas du tout critique pour le relevé de la limite d'absorption et n'affecte pas sa structure. Pour la plupart des échantillons, il existe une absorption résiduelle, due probablement à la diffusion de la lumière incidente par la surface, qui varie d'un échantillon à l'autre, mais en moyenne cette absorption résiduelle est indépendante de la longueur d'onde. Notre évaluation de $\alpha$ est toujours faite en 
retranchant cette absorption résiduelle quand cela est nécessaire. L'erreur relative maximum admise sur $\alpha$ (quelques pour cents) limite les variations significatives du produit $\alpha$ d à l'intervalle $0,3 \leqslant \alpha d \leqslant 5$. Ceci nous conduit à prendre, pour une même composition, plusieurs échantillons d'épaisseurs différentes afin de couvrir la plus large gamme possible sur la variation de $\alpha$ en fonction de l'énergie des photons incidents.

Les échantillons utilisés sont des lames à faces parallèles avec des épaisseurs comprises entre $10 \mathrm{~mm}$ et quelques dizaines de microns. Les échantillons épais (jusqu'à environ $100 \mu$ ) sont obtenus par polissage mécanique, les échantillons très minces (quelques dizaines de $\mu$ ) sont obtenus par attaque chimique.

2.2 RÉSULTATS EXPÉRIMENTAUX. - L'allure générale des courbes d'absorption mesurées à la température ambiante avec les composés vitreux $\mathrm{Ge}_{x} \mathrm{Se}_{1-x}$ $(0<x<0,4)$ est représentée sur la figure 2 . Sur cette

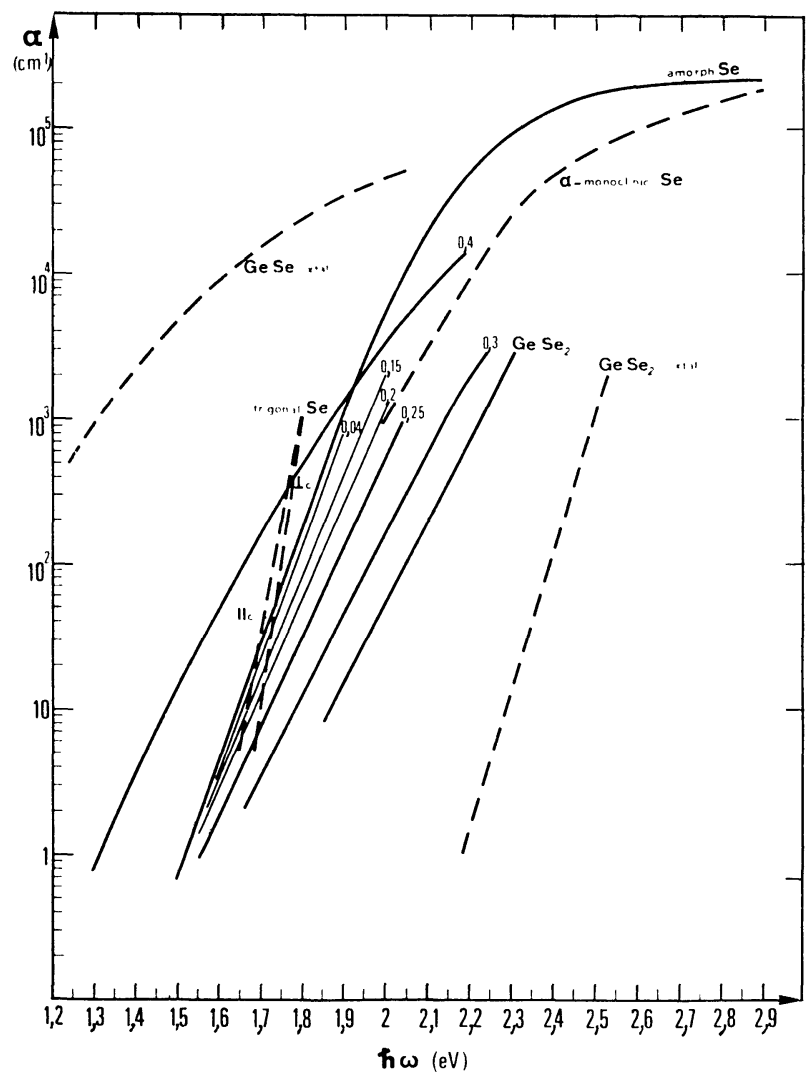

Fig. 2. - Limites d'absorption optique des composés amorphes $\mathrm{Ge}_{x} \mathrm{Se}_{1-x}(0<x<0,4)$ et de $\mathrm{GeSe}_{2}$ monocristal sans polarisation à la température ambiante. Des échantillons d'épaisseurs différentes ont été utilisés $(10 \mathrm{~mm}$ à $20 \mu)$. Les résultats pour différents échantillons de même composition se recouvrent parfaitement. Les courbes de Se trigonal, monoclinique et GeSe monocristallin sont reproduites à partir des références $[8,9,10]$.

figure nous avons également représenté en pointillés les courbes d'absorption du sélénium trigonal, du sélénium monoclinique $\alpha$ et de GeSe cristallin extraites de la littérature $[8,9,10]$, ainsi que nos résultats de mesures pour $\mathrm{GeSe}_{2}$ cristallin en lumière non pola- risée et sous une incidence perpendiculaire au plan de clivage. Lorsque la teneur en germanium croît à partir de $x=0$ la limite d'absorption commence à se déplacer lentement vers les fortes énergies, ensuite le déplacement vers les fortes énergies devient beaucoup plus rapide. La limite d'absorption est enfin déplacée très rapidement vers les faibles énergies lorsqu'on dépasse la valeur $x=1 / 3$.

Dans la gamme des valeurs des coefficients d'absorption mesurés $\left(1 \mathrm{~cm}^{-1}<\alpha<5 \times 10^{3} \mathrm{~cm}^{-1}\right)$ la variation spectrale de $\alpha$ suit pour toutes les compositions une loi exponentielle de la forme :

$$
\alpha=\alpha_{0} \exp \frac{h v}{\Gamma} \mathrm{cm}^{-1}
$$

$h v$ étant l'énergie des photons incidents.

Les valeurs expérimentales de $\alpha_{0}$ et de $\Gamma$ en fonction de $x$ pour $T=300 \mathrm{~K}$ sont données dans le tableau II.

\section{TABleau II}

Paramètres des lois exponentielles décrivant les limites d'absorption dans les composés $\mathrm{Ge}_{x} \mathrm{Se}_{1-x}$ $0 \leqslant x \leqslant 1 / 3$ à la température ambiante.

\begin{tabular}{ccc}
\multicolumn{3}{c}{$\alpha=\alpha_{0} \exp \frac{h v}{\Gamma} \mathrm{cm}^{-1}$} \\
Composition & $\alpha_{0} \mathrm{~cm}^{-1}$ & $\Gamma \mathrm{meV}$ \\
$\frac{\mathrm{Se}}{\mathrm{Ge}_{0,04} \mathrm{Se}_{0,96}}$ & $1,6 \times 10^{-12}$ & $56 \pm 2$ \\
$\mathrm{Ge}_{0,10} \mathrm{Se}_{0,90}$ & $3,1 \times 10^{-12}$ & $58 \pm 2$ \\
$\mathrm{Ge}_{0,15} \mathrm{Se}_{0,85}$ & $5,1 \times 10^{-11}$ & $64 \pm 2$ \\
$\mathrm{Ge}_{0,20} \mathrm{Se}_{0,80}$ & $1,9 \times 10^{-10}$ & $68 \pm 2$ \\
$\mathrm{Ge}_{0,25} \mathrm{Se}_{0,75}$ & $1,4 \times 10^{-8}$ & $75 \pm 3$ \\
$\mathrm{Ge}_{0,30} \mathrm{Se}_{0,70}$ & $5,9 \times 10^{-9}$ & $77 \pm 3$ \\
$\mathrm{GeSe}_{2}$ amorphe & $6,0 \times 10^{-10}$ & $78 \pm 3$
\end{tabular}

$\Gamma$ croît en fonction de $x$, il en est de même pour $\alpha_{0}$ mais la précision relative sur l'évaluation de ce dernier paramètre est très mauvaise $\left(\Delta \alpha_{0} / \alpha_{0} \simeq 50 \%\right)$. Nos résultats dans le sélénium vitreux sont en accord avec ceux de Kartke et Regensburger [11] qui trouvent

$$
\alpha=7,35 \times 10^{-12} \exp (h v / 0,058)\left(\mathrm{cm}^{-1}\right) .
$$

2.3 ANALYSE DES RÉSUltats. - La figure 3 représente les limites d'absorption optique à la température ambiante de $\mathrm{Ge}$ [12], Se [13] et $\mathrm{GeSe}_{2}$ dans leurs phases vitreuses et cristallines. D'après les positions relatives en énergie de ces limites d'absorption, la présence dans un composé $\mathrm{GeSe}_{1-x}$ de liaisons Ge-Ge (agglomérats de germanium type germanium amorphe) apparaît comme étant l'élément structural déterminant pour l'absorption optique dans la gamme spectrale étudiée : 


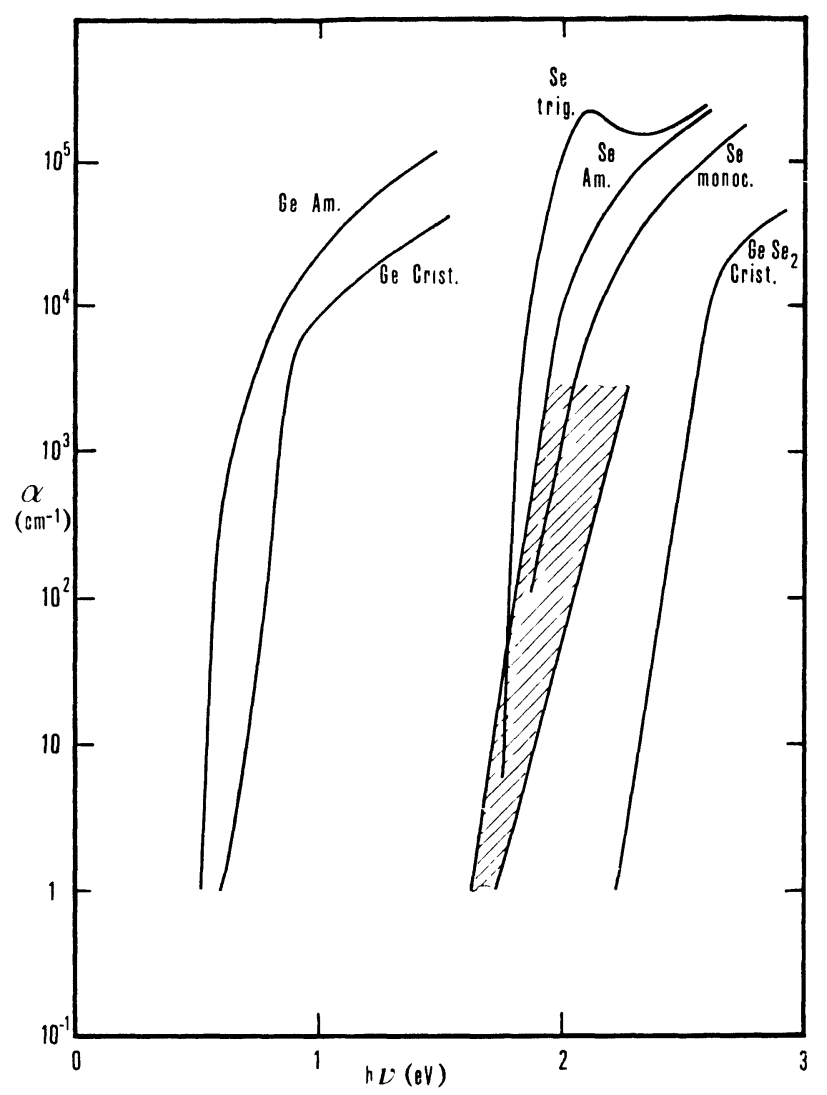

Fig. 3. - Limites d'absorption optique de Ge référence [12]. Se référence [13] et $\mathrm{GeSe}_{2}$ (amorphes et cristallins). Les composés amorphes $\operatorname{Ge}_{x} \mathrm{Se}_{1-x}(0 \leqslant x \leqslant 1 / 3)$ présentent une absorption (zone hachurée) comprise entre celles de $\mathrm{Se}$ et de $\mathrm{GeSe}_{2}$.

pour $h v=1 \mathrm{eV}$, le coefficient d'absorption $\alpha$ dans Ge est de l'ordre de $10^{4} \mathrm{~cm}^{-1}$ alors que dans $\mathrm{Se}$ et $\mathrm{GeSe}_{2}$ $\alpha$ est pratiquement nul (non mesurable). Les composés vitreux $\mathrm{Ge}_{x} \mathrm{Se}_{1-x}$ pour $0 \leqslant x \leqslant 1 / 3$ (on se limitera au cours de cette discussion à cette gamme de composition), présentent tous à énergie de photon égale une absorption optique comprise entre celle du sélénium amorphe et celle de $\mathrm{GeSe}_{2}$ cristallin. Ce résultat montre que les composés $\mathrm{Ge}_{x} \mathrm{Se}_{1-x}$ ne sont pas constitués d'agglomérats de germanium d'une part et de sélénium d'autre part. Les atomes de germanium se diluent au maximum dans le sélénium pour former un réseau avec des configurations qui s'apparentent à celles existant dans les composés extrêmes $\mathrm{Se}$ et $\mathrm{GeSe}_{2}$. Les propriétés optiques d'un tel réseau ne sont pas faciles à prévoir par des considérations théoriques. Aussi nous allons tenter de dégager, par une analyse qualitative, la relation entre les limites d'absorption et les configurations locales d'atomes dans ces composés.

2.3.1 Comportement exponentiel de la limite d'absorption. - Nous avons observé dans tous les composés étudiés (vitreux et cristallins) dans la gamme $10 \mathrm{~cm}^{-1}<\alpha<5 \times 10^{3} \mathrm{~cm}^{-1}$, une dépendance spectrale exponentielle de la limite d'absorption. Un tel comportement de la limite d'absorption a été observé dans de nombreux matériaux [14] aussi bien vitreux que cristallins. Le paramètre $\Gamma$ est toujours de l'ordre de $20 \mathrm{meV}$ à $100 \mathrm{meV}$ à la température ambiante [15]. Malgré l'uniformité et la convergence dans les caractéristiques principales de cette propriété physique quel que soit le matériau, on peut considérer que jusqu'à présent il n'existe pas de théorie complète et unique permettant d'en donner une interprétation satisfaisante [14]. Néanmoins de nombreux auteurs [16] s'accordent pour attribuer l'élargissement du seuil fondamental optique à la présence d'impuretés ou de défauts (queues de densité d'états dues à des fluctuations de potentiel local).

Nous avons porté les valeurs du paramètre $\Gamma$ en fonction de la composition (Fig. 4) on obtient une

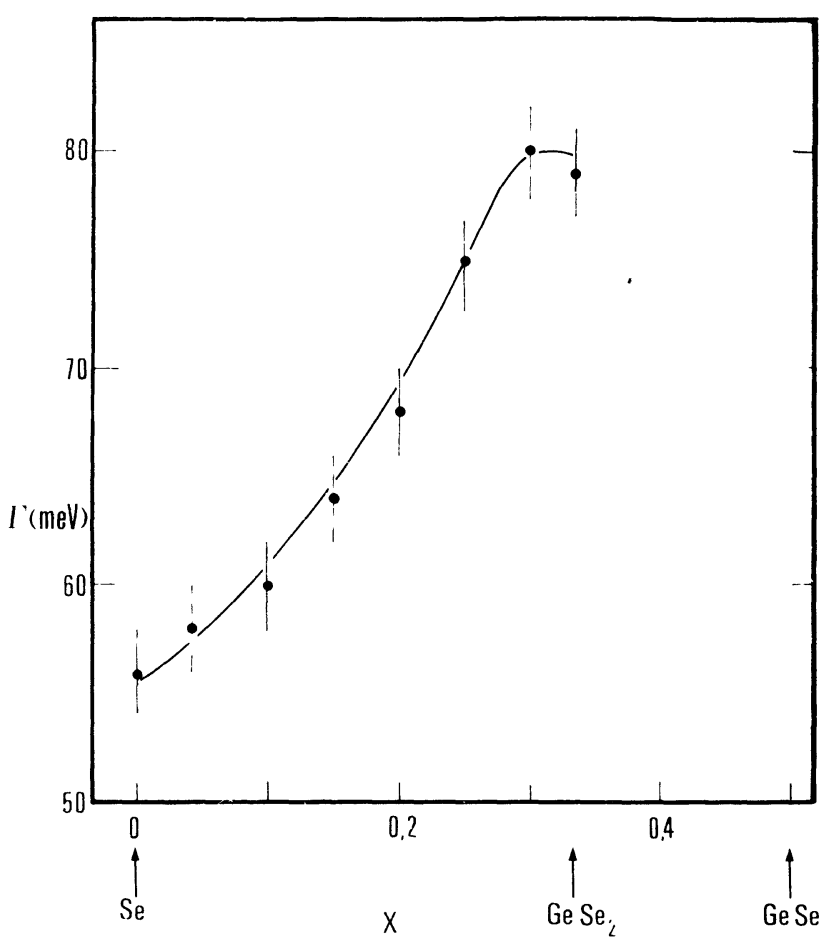

Fig. 4. - Variation du paramètre $\Gamma$ (inverse de la pente de la limite d'absorption) en fonction de la composition des composés amorphes $\mathrm{Ge}_{x} \mathrm{Se}_{1-x}(0 \leqslant x \leqslant 1 / 3)$.

courbe moyenne qui présente un maximum pour $\mathrm{GeSe}_{2}$ vitreux. Un tel comportement traduit probablement un degré de désordre local variable suivant la composition. L'origine de ce désordre est à relier au mode de préparation, et aux propriétés mécaniques de ces matériaux. D'une part, pour des teneurs en germanium supérieures à $x=0,20$ on doit effectuer lors de la préparation une trempe rapide du mélange liquide afin d'éviter la cristallisation. La vitesse de trempe nécessaire passe par un maximum pour la composition $\mathrm{GeSe}_{2}$. Les contraintes internes dues à cette trempe augmentent donc avec $x$. D'autre part, la microdureté ainsi que la température de ramollissement $\left(T_{\mathrm{g}}\right)$ [6] croissent en fonction de $x$. Ce durcissement des composés au fur et à mesure que la teneur en germanium augmente, correspond probablement au passage 
d'une structure moléculaire composée de chaînes et d'anneaux (sélénium pur) à une structure faite de motifs tétraédriques $\left(\mathrm{GeSe}_{4}\right)$ possédant des liaisons dans les trois dimensions. Cette progression d'un réseau unidimensionnel vers un réseau tridimensionnel crée certainement des tensions internes locales supplémentaires (d'autant plus que $\mathrm{GeSe}_{2}$ cristallin est un composé lamellaire). Il faut noter enfin que des fluctuations locales de potentiel peuvent également résulter de la différence d'électronégativité entre le sélénium et le germanium. Quand la teneur en germanium croît le nombre de liaisons à caractère partiellement ionique augmente. Il semble difficile pour l'instant de donner une description quantitative de ces effets en termes de champs électriques internes. Il faut souligner toutefois que les fluctuations de pentes ne masquent pas l'effet de translation des limites d'absorption en fonction de $x$, qui reste l'effet principal.

2.3.2 Seuil fondamental optique. - Dans tout semiconducteur l'étude de la limite d'absorption permet de déterminer un seuil fondamental optique $E_{0} . E_{0}$ correspond à la largeur de la bande interdite $E_{\mathrm{g}}$ (Gap) entre le minimum le plus bas en énergie de la bande de conduction et le maximum le plus haut en énergie de la bande de valence dans un modèle d'excitation à un électron [7]. Sans entrer dans le détail des mécanismes d'absorption optique responsables de la dépendance spectrale d'une limite d'absorption, on peut, comme cela est suggéré par de nombreux auteurs [17], adopter une valeur arbitraire du coefficient d'absorption pour définir $E_{0}$. Généralement on prend $\alpha$ dans la gamme $10^{2} \mathrm{~cm}^{-1} \leqslant \alpha \leqslant 10^{4} \mathrm{~cm}^{-1}$. On considère que ces valeurs de $\alpha$ caractérisent le début des transitions bande à bande en dehors de tout processus d'absorption dû à des états localisés dans la bande interdite (impuretés, défauts). Nous avons porté sur la figure 5 les courbes d'absorption en fonction de la composition pour quatre valeurs de $\alpha\left(10^{3} \mathrm{~cm}^{-1}, 5 \times 10^{2} \mathrm{~cm}^{-1}\right.$, $10^{2} \mathrm{~cm}^{-1}$ et $10 \mathrm{~cm}^{-1}$ ). Pour $0 \leqslant x \leqslant 1 / 3$ toutes ces courbes indiquent une cassure à $x=0,20$. On voit apparaître ainsi une corrélation entre la variation de $E_{0}$ avec $x$ et celle de l'indice de réfraction $n$ ou du spectre Raman avec ce même paramètre ; or les dépendances en $x$ du spectre Raman puis celles de $n$ ont pu être interprétées par référence aux types de liaisons possibles dans cette gamme de composition. Ces résultats suggèrent donc que dans tout calcul théorique des niveaux énergétiques permis pour les électrons de valence et de conduction dans les composés $\mathrm{Ge}_{x} \mathrm{Se}_{1-x}(0 \leqslant x \leqslant 1 / 3)$ il faut faire intervenir au moins les premiers voisins de chaque atome. En effet, si on ne considère comme White [18] que la liaison chimique, entre Se et $\mathrm{GeSe}_{2}$ on ne peut identifier que deux types de liaisons: Se-Se (type sélénium) et $\mathrm{Ge}-\mathrm{Se}$ (type $\mathrm{GeSe}_{2}$ coordinance 4-2). Ceci ne fait pas apparaître de composé intermédiaire particulier. Par contre, si on prend en compte les premiers voisins, on a (dans la mesure ou on suppose toujours

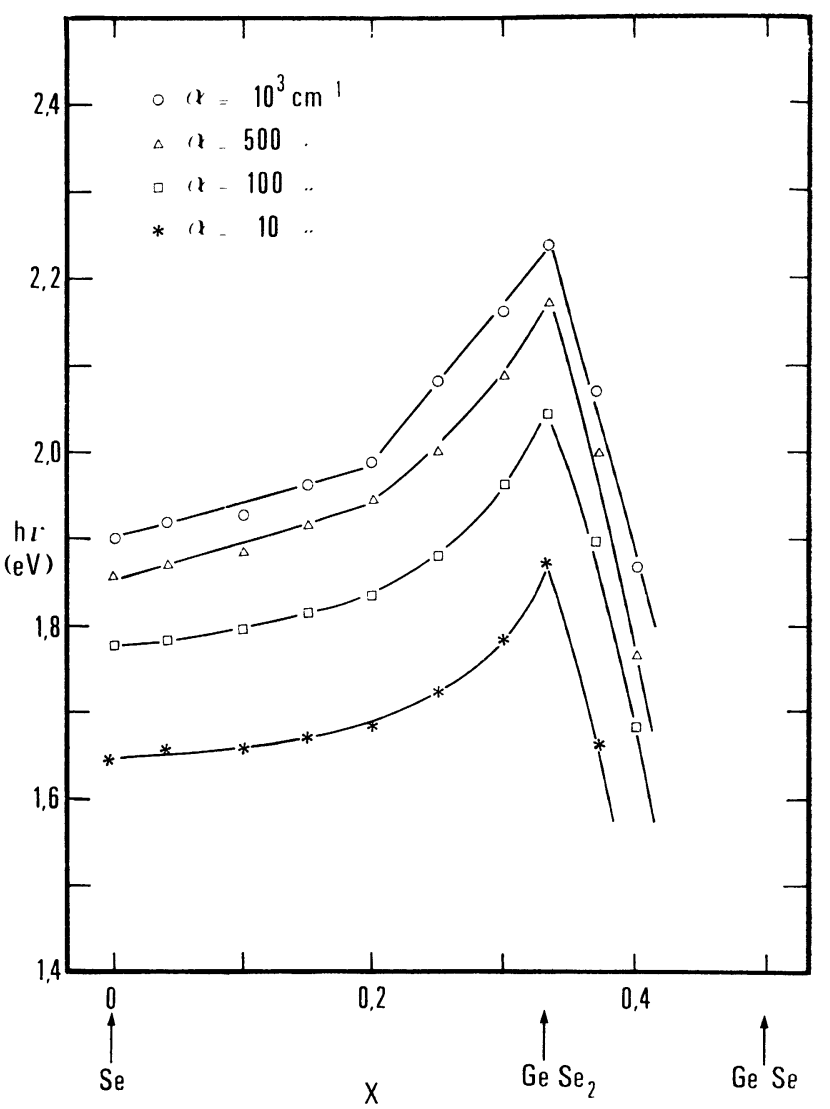

Fig. 5. - Variation du seuil fondamental optique en fonction de la composition pour les composés amorphes $\mathrm{Ge}_{x} \mathrm{Se}_{1-x}$ $(0 \leqslant x \leqslant 0,4)$. A partir de ces courbes on montre l'existence d'une corrélation entre le comportement de la limite d'absorption en fonction de $x$ et l'ordre local dans ces composés.

une dilution parfaite des atomes de germanium dans le sélénium avec respectivement une coordinance 4 et 2) pour $0 \leqslant x \leqslant 0,20$ deux types de sites sélénium : $\mathrm{Se}-\mathrm{Se}-\mathrm{Se}$ (Se pur) et $\mathrm{Ge}-\mathrm{Se}-\mathrm{Se}\left(\mathrm{GeSe}_{4}\right)$; et pour $0,20 \leqslant x \leqslant 1 / 3$ également deux types de sites sélénium : Ge-Se-Se $\left(\mathrm{GeSe}_{4}\right)$ et $\mathrm{Ge}-\mathrm{Se}-\mathrm{Ge}\left(\mathrm{GeSe}_{2}\right)$. Dans un tel réseau on a effectivement disparition à partir de $x=0,20$ de sites sélénium dans un environnement purement sélénium.

Il est intéressant de comparer ces résultats à ceux obtenus dans d'autres séries de composés semiconducteurs. Les résultats les plus précis et les mieux compris existant dans la littérature concernent des alliages de semiconducteurs cristallins. Une variation linéaire du seuil fondamental en fonction de la composition indique que les structures de bandes des cristaux constituant l'alliage peuvent présenter des analogies [19]. Si les structures de bandes des composés extrêmes de la série ne présentent aucune parenté, un changement abrupt se produit sur la courbe $E_{0}(x)$ à une composition intermédiaire [20]. Dans le premier cas, la structure cristalline des composés extrêmes de la série est identique, l'évolution de $E_{0}$ en fonction de la composition est due à une variation des distances interatomiques. Dans le second cas la structure cristalline des 
composés extrêmes n'est pas identique et la variation de composition conduit à une distorsion de l'environnement local. Il n'existe pas d'investigations semblables dans les alliages amorphes. Cependant de nombreux auteurs [5] ont trouvé des corrélations entre $E_{0}$ et la température de fusion ou la température de ramollissement $T_{\mathrm{g}}$ dans de nombreux composés. Ces corrélations sont interprétées par la coordinance moyenne des atomes. Cette approche est intéressante mais n'a pas de fondement théorique.

Dans les composés $\mathrm{Ge}_{x} \mathrm{Se}_{1-x}(0 \leqslant x \leqslant 1 / 3)$ il est bien établi [2] que d'une part la coordinance des atomes (4-2) ainsi que la distance interatomique moyenne $(2,3 \AA$ à $2,4 \AA)$ sont pratiquement indépendantes de $x$, d'autre part la densité varie très peu avec $x(\simeq 2 \%)$. Par ailleurs [6] la température de ramollissement $\left(T_{\mathrm{g}}\right)$ varie de façon monotone avec $x$ dans cette gamme de composition. Il est clair donc que la variation de $E_{0}$ avec $x$ est essentiellement reliée à la configuration locale des atomes dans ces composés.

En résumé il apparaît que le paramètre $\Gamma$ est sensible à l'importance des distorsions de l'ordre à courte distance liées probablement aux défauts et aux contraintes dus au mode de préparation, contrairement à la limite d'absorption $E_{0}(x)$ qui traduit la nature des liaisons chimiques et de leur environnement local.

Pour $x>1 / 3$ les résultats expérimentaux sont trop partiels pour permettre une démarche analogue à la précédente. On peut toutefois attribuer la décroissance rapide de $E_{0}$ au-delà de $x=1 / 3$ (Fig. 5) soit à la possibilité de coordination des atomes de germanium entre eux soit à l'apparition d'une structure locale du type GeSe cristallin avec changement de coordinance des atomes de germanium et de sélénium [21].

3. Conclusion. - L'étude de l'indice de réfraction et de la limite d'absorption permet d'étayer le modèle, de structure atomique locale et de son évolution avec la teneur en germanium dans les composés $\mathrm{Ge}_{x} \mathrm{Se}_{1-x}$ pour $0 \leqslant x \leqslant 0,33$, établi à partir de mesures de spectrométrie Raman. Il apparaît en effet d'après nos mesures une étroite corrélation des variations du coefficient d'absorption, de l'indice de réfraction avec $x$ et celle du spectre Raman avec ce même paramètre. Ce résultat est interprété par référence aux types de liaison possibles dans ces composés : absence de liaison $\mathrm{Ge}-\mathrm{Ge}$, coordinance 4-2 respectivement pour les atomes de germanium et de sélénium, les séquences $\mathrm{Ge}-\mathrm{Se}-\mathrm{Ge}$ restent peu nombreuses dans la mesure où la teneur en germanium le permet. Enfin les pentes des limites d'absorption présentent une évolution avec la composition qui caractérise le degré de distorsion lié à l'importance des défauts et des contraintes induites par la tendance plus ou moins grande à la cristallisation.

\section{Bibliographie}

[1] Tronc, P., Bensoussan, M., Brenac, A., Sebenne, C., Phys. Rev. B 8 (1974) 5947.

[2] Poltavtsev, Y. G., Pozdnayakova, Inorg. Mat. 9 (1973) 766.

Malaurant, J., J. Non. Cryst. Solids, à paraître.

Fawcett, R. W., WaGner, C. N. J., Cargill III, G. S., J. Non. Cryst. Solids 8-10 (1972) 369.

Uemura, O., Sagara, Y., Salotow, T., Phys. Stat. Sol. (a) 26 (1974) 99.

SaPre, V. B., Mande, C., Indian J. Pure Appl. Phys. 12 (1974) 74

Sayers, D. E., Lytle, F. W., Proceed. of 5 th Int. Conf. on Amorphious and liquid Semicond. (éditeur Taylor et Francis London) 1974 p. 403.

[3] Hilton, A. R., Jones, C. E., Appl. Opt. 6 (1967) 1513.

[4] Kastner, M., Phys. Rev. B 6 (1972) 2273.

[5] KAStner, M., Phys. Rev. B 7 (1973) 5237.

[6] Azoulay, R., Thibierge, H., Brenac, A., J. Non. Cryst. Solids 18 (1975) 33.

[7] Tauc, J., " Progress in Semiconductors » (Heywood Bouh) 1965 Vol. 9 p. 87.

[8] Henrion, W., Physics of Selenium and Telurium édité par Cooper W. C. (Pergamon Press Oxford) 1959 p. 75.

[9] Knights, J. C., Davis, E. A., Solid State Commun. 11 (1972) 543.
[10] Kannewurf, C. R., Cashman, R. F., J. Phys. Chem. Solids 22 (1961) 293.

[11] Hartke, J. L., Regensburger, P. J., Phys. Rev. 139 (1965) 970.

[12] Stuke, J., J. Non. Cryst. Solids 4 (1970) 1.

[13] Davis E. A., Electronic and Structural Properties of Amorphous Semiconductors (édité par Le Comber P. G. et Mort J. Academic Press) 1973, p. 425.

[14] KurIK, M. V., Phys. State Solid. 8 (1971) 9.

[15] Chopra, K. L., BaHL, S. K., Thin Solid Films 11 (1972) 377.

[16] Dow, J. D., Redfield, D., Phys. Rev. B 1 (1970) 3358.

[17] Mott, N. F., Davis, E. A., "Electronic Progresses in Non-crystalline Materials » (Clarendon Press Oxford 1971).

[18] White, R. M., Proceed. of 5th Int. Conf. on Amorphous and Liquid Semicond. (éditeur Taylor et Francis London 1974) p. 1081.

[19] LoNG, D., "Semiconductors and Semi-metals » (édité par Willardson K. R. et Beer A. C., Academic Press New York) 1966 Vol. 1 p. 143.

[20] Long, D., J. Appl. Phys. 33 (1962) 1682.

[21] Tronc, P., Bensoussan, M., Brenac, A., Errandonea, G., Sebenne, C., Phys. Rev. (à paraître). 